\title{
Diagnosis of Growth Hormone Deficiency Remains a Judgment Call - and That Is Good
}

\author{
David B. Allen \\ Department of Pediatrics, University of Wisconsin School of Medicine and Public Health, Madison, WI, USA
}

\section{Keywords}

False positive · Growth hormone testing

\begin{abstract}
The diagnosis of growth hormone deficiency (GHD) still does not reflect evidence-based and generally accepted practice, and reliance on growth hormone stimulation testing (GST) leads to a high rate of false-positive diagnosis of idiopathicisolated GHD (IIGHD). While searching for more definitive indicators of GHD is attractive, it should not distract from currently available steps to reduce erroneous IIGHD diagnoses. This paper describes opportunities to improve the accuracy of the GST which include: (1) meticulous selection of candidates for GST, since a low prevalence of GHD among short children in general is a major factor undermining the test's diagnostic accuracy; (2) departure from traditional pass/fail diagnostic GH cutoffs toward, instead, formulation of diagnoses along a continuum that spans actual GHD - > provisional GHD - > not GHD; (3) response to the provisional diagnosis of IIGHD based on GST with additional post-test observation or alternative growth-promoting interventions rather than immediate human growth hormone treatment; (4) re-examination and often correction of a prior IIGHD diagnosis with the onset of puberty. Modern medicine is increasingly offering diagnostic tests that aim to eliminate the need for provisional diagnoses. But a pitfall of such a "definitive" test for GHD would be the temptation to respond to its results definitively. Given the nuances, variations, and fluctuations in $\mathrm{GH}$ axis function over time, children evaluat-
\end{abstract}

karger@karger.com

(c) 2021 S. Karger AG, Basel

www.karger.com/hrp

Karger" ed for growth concerns are still best served by clinical judgment that combines thoroughness, patience, flexibility, and healthy skepticism into the diagnosis of GHD.

(c) 2021 S. Karger AG, Basel

More than a half century since the advent of treatment for growth hormone deficiency (GHD), the diagnosis of GHD still does not reflect evidence-based and generally accepted practice. Recently in Hormone Research in Paediatrics, Bright et al. [1] described mathematical shortcomings of GH-stimulation testing (GST) in diagnosing GHD, namely, that the rarity of the condition combined with test imprecision lead to an estimate that, in short children undergoing GST, a false-positive diagnosis of GHD is made in 35/36 instances [1]. The focus on overdiagnosis of GHD is appropriate, since the under-diagnosis of GHD - in particular failure to detect severe GHD in need of prompt treatment - is not a significant problem. Children with severe GHD due to anatomic abnormalities, established genetic causes of GHD, or mutations in transcription factors causing multiple pituitary hormone deficiency nearly always show stimulated GH concentrations clearly distinct from the normal range $[2,3]$. The subgroup of diagnostic uncertainty and controversy is idiopathic-isolated GHD (IIGHD), most often identified in mid-to-late childhood based on (non-sex steroid primed) GST. Without much explanation, the authors theorize that identification of enrichment marker predictors of positive growth response to recombinant human 
growth hormone (hGH) would improve diagnostic accuracy of GHD.

While searching for more definitive indicators of GHD is attractive, it should not distract from steps that can already be taken to improve the value of GST in making a clinical diagnosis. An alternate perspective contends that (1) no test will clearly and consistently distinguish normal from abnormal function of a system that is intrinsically pulsatile, varies with age and puberty stage and body composition, and depends on a continuum of layered stimulation, suppression, and end-organ response to regulate linear growth; and (2) accuracy of the GST is improved by judicious selection of patients to test, critical interpretation of results, and responses to these interpretations that incorporate awareness of the test's limitations in both the short and longer term.

A first opportunity to avoid an erroneous diagnosis of GHD is meticulous selection of candidates for GST, since a low prevalence of GHD among short children in general is a major factor undermining the test's diagnostic accuracy. A high GST false-positive rate can be reduced by limiting testing to children with pre-test clinical and laboratory features that strongly point to GHD as a likely cause of disordered growth: those with slow growth rate and growth pattern atypical for family, delayed bone age, absence of systemic illness or use of growth-inhibiting medications or suggestive syndromic characteristics, and normal preliminary laboratory screening for other growth-limiting problems. Recognizing and assigning appropriate importance to positive and negative clues for GHD are key steps in estimating its pre-test probability for an individual child [4]. For children whose clinical picture points toward GHD, measurement of IGF-1 and (in young children) IGFBP-3 levels can augment estimates of GHD likelihood [4]. However, variations in IGF-1 with age, sex, pubertal status, and body mass index, and lack of robust data guiding accurate adjustments for these variations complicates identification of threshold levels for IGF-1 and IGFBP-3 that reliably predict or rule out GHD [5]. Based on individual [6] and metanalysis [7] studies, an IGF-I cutoff at -2 SDS has sensitivity of $\sim 65 \%$ and specificity $\sim 80 \%$ for subsequent diagnosis of GHD by GST. For IGFBP-3, a cutoff at -2 SDS yields similar sensitivity (50\%) and specificity (80\%), with a low IGFBP-3 being less sensitive but more specific than a low IGF-I as an indicator of GHD [6, 7]. These data suggest that $\sim 35 \%$ of children with GHD will have an IGF- 1 level higher than -2 SD so that such cutoffs cannot be adhered to tightly to avoid under-diagnosis of GHD in children with high clinical pre-test likelihood. On the other hand, higher IGF-1 levels can help in selection of appropriate children for GST, since there is low and very low likelihood of GHD when IGF-1 levels are higher than -1 SD (14\%) or 0 SD (4\%) respectively [6]. A notable exception is cancer-surviving children, in whom GST should be performed whenever there is clinical suspicion of GHD, since a low serum IGF-I has low sensitivity as an indicator of GHD in such children [8]. In summary, even with the limitations noted above, careful analysis of prior clinical and historical clues combined with IGF-1/ IGFBP-3 results should allow exclusion of a large proportion of short-statured children from GST.

Once GST is decided upon, a second opportunity to minimize false-positive diagnosis of GHD is to resist adherence to traditional pass/fail diagnostic GH cutoffs and instead interpret results to formulate a diagnosis along a continuum that spans actual GHD - > provisional GHD - > not GHD. Regarding traditional GST diagnostic thresholds for GHD (generally 7-10 $\mu \mathrm{g} / \mathrm{L}$ ), modern immunometric methods reveal that $10 \mu \mathrm{g} / \mathrm{L}$ is just below the mean response and $<5 \mu \mathrm{g} / \mathrm{L}$ to be at the 5 th percentile in normally growing children undergoing GST [9]. Consequently, as endorsed by recent guidelines, it is reasonable to distinguish severe (i.e., actual) GHD in children with a very low GST peak GH level of (e.g.) $<3 \mu \mathrm{g} / \mathrm{L}$ from partial GHD (i.e., termed "provisional" here because test result is consistent with but not diagnostic of GHD) in children with a GST peak GH level of (e.g.) 5-10 $\mu \mathrm{g} / \mathrm{L}$ [10]. Since no precise cutoff level can definitively separate diagnostic groups, placement of a child with intermediate results (e.g.) $3-5 \mu \mathrm{g} / \mathrm{L}$ into the "actual" versus "provisional" GHD group should reflect pre-test likelihood based on clinical judgment and ancillary studies (e.g., MRI). The salient point is that replacing the standard dichotomous positive/negative interpretation of GST results with a more flexible patient-specific approach can reduce falsepositive diagnosis of GHD if children deemed provisionally GHD become candidates for alternative non-hGH diagnostic-therapeutic interventions.

This situation arises commonly in the care of short children with constitutional delay of growth and puberty (CDGP), who typically show downward crossing of height percentile lines in late childhood [11] and often a reduced GH response to GST [12]. Growth hormone responses to GST are similarly blunted in obese or overweight individuals proportionate to increase in body mass index [13]. Sex steroid priming of prepubertal children (boys $>11$ years and girls $>10$ years with adult height prognosis within $-2 \mathrm{SD}$ of the reference population mean) prior to GST can provide results that more accu- 
rately indicate the child's capacity to produce GH during puberty, reduce laboratory diagnosis of GHD and MRI scans ordered to rule out pathology, and prevent unnecessary hGH treatment [10]. Critics of pre-GST sex steroid priming argue that simulating puberty does not allow discovery of the cause of the child's slow growth at that time - which may in fact reflect reduced prepubertal GH production. Therefore, if non-sex steroid-primed GST is performed in peri-pubertal children with CDGP or obesity, low GH levels should be considered only provisionally diagnostic of GHD, and prompt subsequent steps that may correct misdiagnosis and avoid unnecessary hGH treatment.

Specifically, a third opportunity to limit the over-diagnosis of GHD is to respond to the provisional diagnosis of GHD with actions other than immediate hGH treatment [14]. For the vast majority of short children in this group - in whom the true diagnoses are predominantly childhood IIGHD, ISS, and CDGP - a low GH response to GST may justify an MRI of the hypothalamus-pituitary area to exclude pathology but does not necessitate prompt prescription of hGH. Following a reassuring normal MRI, approaches that corroborate or refute the diagnosis (and are often cost-effective) can be considered as alternatives to simply "confirming" a presumed GHD diagnosis by showing a response to hGH [15]. For toddlers and school-aged children, in whom partial GHD versus CGDP is the most common diagnostic dilemma, observation for an additional 3-6 months to assess whether slow growth persists can clarify for prescriber and family alike whether hGH replacement is actually needed. As a personal observation, the author has seen several such children "defy" GST results that suggest partial GHD with subsequent normal growth. For older children, a useful diagnostic/therapeutic alternative prior to resorting to $\mathrm{hGH}$, and one that may clarify the transient nature of the prepubertal GHD, is very low-dose oral oxandrolone (e.g., 0.625-1.25 mg per day) [16], though unfortunately not FDA-approved for growth acceleration and not available in all countries. Oxandrolone (which does not increase endogenous GH secretion) typically increases growth rate by $3-5 \mathrm{~cm}$ per year $[17,18]$ and provides a useful growth-sustaining "bridge" to the onset of puberty, at which time the child's growth response to an endogenously sex steroid-primed $\mathrm{GH}$ axis can be evaluated. In peri-pubertal children whose diagnosis of GHD is based on a non-sex steroid-primed GST and whose skeletal age is suitably mature, an increase in growth rate and IGF-1 levels in response to a short-term trial of low-dose testosterone or estrogen will clarify the transient nature of the prepubertal GHD diagnosis.
A final opportunity to limit the persistence of a falsepositive diagnosis of GHD is to subsequently question and re-examine the diagnosis. For a strong majority of children diagnosed with IIGHD based on GST but without identifiable hypothalamic-pituitary abnormalities, normal GH secretion is observed after puberty onset [19]. Thus, retesting of the hypothalamic-pituitary-GH/IGF-I axis when a child treated for IIGHD enters puberty will, in most cases, reveal a normal GH peak in response to GST $[20,21]$. In spite of this evidence, it remains predominant practice to sustain diagnosis and treatment of IIGHD until epiphyseal closure. An alternative approach is to interrupt hGH treatment at puberty onset for IIGHD children who have a height prognosis within the low normal range. This is followed by careful growth rate observation and IGF-1 measurement, with retesting for IIGHD if IGF-1 levels decline markedly and/or reinstitution of treatment if persistent slow growth occurs [15].

Admittedly, the diagnosis of GHD is challenging and the GST is far from a definitive diagnostic tool. However, as described above, when careful analysis of clinical and laboratory data guide not only the decision to perform a GST, but also guide a patient-specific interpretation, therapeutic response to, and subsequent re-examination of provisionally diagnostic results, erroneous diagnosis of GHD can be significantly reduced in both frequency and duration. Bright et al. [1] highlight a tension in modern medicine created by the search for diagnostic tests that aim to eliminate the need for provisional diagnoses. But a pitfall of such a "definitive" test for GHD would be the temptation to respond to its results definitively. Given the nuances, variations, and fluctuations in $\mathrm{GH}$ axis function over time, children evaluated for growth concerns are still best served by clinical judgment that combines thoroughness, patience, flexibility, and healthy skepticism into the diagnosis of GHD.

\section{Conflict of Interest Statement}

Dr. Allen has received an honorarium from BioMarin.

\section{Funding Sources}

No funding was received.

\section{Author Contributions}

The author designed and wrote the entire commentary on his own. 


\section{References}

1 Bright GM, Morris PA, Rosenfeld RG. When is a positive test for pediatric growth hormone deficiency a true positive test? Horm Res Paediatr. 2021.

2 Coutant R, Rouleau S, Despert F, Magontier $\mathrm{N}$, Loisel D, Limal JM. Growth and adult height in GH-treated children with nonacquired GH deficiency and idiopathic short stature: the influence of pituitary magnetic resonance imaging findings. J Clin Endocrinol Metab. 2001;86(10):4649-54.

3 Maghnie M, Lindberg A, KoltowskaHäggström M, Ranke MB. Magnetic resonance imaging of CNS in 15,043 children with GH deficiency in KIGS (Pfizer international growth database). Eur J Endocrinol. 2013; 168(2):211-7.

4 Wit JM, Bidlingmaier M, de Bruin C, Oostdijk W. A proposal for the interpretation of serum IGF-I concentration as part of laboratory screening in children with growth failure. J Clin Res Pediatr Endocrinol. 2020;12(2): 130-9.

5 Blum WF, Alherbish A, Alsagheir A, El Awwa A, Kaplan W, Koledova E, et al. The growth hormone-insulin-like growth factor-I axis in the diagnosis and treatment of growth disorders. Endocr Connect. 2018;7(6):R212-r222.

6 Rikken B, van Doorn J, Ringeling A, Van den Brande JL, Massa G, Wit JM. Plasma levels of insulin-like growth factor (IGF)-I, IGF-II and IGF-binding protein-3 in the evaluation of childhood growth hormone deficiency. Horm Res. 1998;50(3):166-76.

7 Shen Y, Zhang J, Zhao Y, Yan Y, Liu Y, Cai J. Diagnostic value of serum IGF-1 and IGFBP-3 in growth hormone deficiency: a systematic review with meta-analysis. Eur J Pediatr. 2015;174(4):419-27.
8 Sklar CA, Antal Z, Chemaitilly W, Cohen LE, Follin C, Meacham LR, et al. Hypothalamicpituitary and growth disorders in survivors of childhood cancer: an endocrine society clinical practice guideline. J Clin Endocrinol Metab. 2018;103(8):2761-84.

9 Ghigo E, Bellone J, Aimaretti G, Bellone S, Loche S, Cappa M, et al. Reliability of provocative tests to assess growth hormone secretory status. Study in 472 normally growing children. J Clin Endocrinol Metab. 1996;81(9): 3323-7.

10 Grimberg A, DiVall SA, Polychronakos C, Allen DB, Cohen LE, Quintos JB, et al. Guidelines for growth hormone and insulin-like growth factor-I treatment in children and adolescents: growth hormone deficiency, idiopathic short stature, and primary insulin-like growth factor-I deficiency. Horm Res Paediatr. 2016;86(6):361-97.

11 Butenandt O, Kunze D. Growth velocity in constitutional delay of growth and development. J Pediatr Endocrinol Metab. 2010; 23(1-2):19-25.

12 Saggese G, Cesaretti G, Giannessi N, Bracaloni C, Cinquanta L, Cioni C. Stimulated growth hormone $(\mathrm{GH})$ secretion in children with delays in pubertal development before and after the onset of puberty: relationship with peripheral plasma GH-releasing hormone and somatostatin levels. J Clin Endocrinol Metab. 1992;74(2):272-8.

13 Argente J, Caballo N, Barrios V, Pozo J, Muñoz MT, Chowen JA, et al. Multiple endocrine abnormalities of the growth hormone and insulin-like growth factor axis in prepubertal children with exogenous obesity: effect of short- and long-term weight reduction. J Clin Endocrinol Metab. 1997;82(7):2076-83.
14 Wit JM, Oostdijk W. Novel approaches to short stature therapy. Best Pract Res Clin Endocrinol Metab. 2015;29(3):353-66.

15 Allen DB. Cost-conscious growth-promoting treatment: when discretion is the better part of value. Horm Res Paediatr. 2018;90(3):14550.

16 Allen DB, Cuttler L. Clinical practice. Short stature in childhood-challenges and choices. N Engl J Med. 2013;368(13):1220-8.

17 Salehpour S, Alipour P, Razzaghy-Azar M, Ardeshirpour L, Shamshiri A, Monfared MF, et al. A double-blind, placebo-controlled comparison of letrozole to oxandrolone effects upon growth and puberty of children with constitutional delay of puberty and idiopathic short stature. Horm Res Paediatr. 2010; 74(6):428-35.

18 Schroor EJ, van Weissenbruch MM, Knibbe P, Delemarre-van de Waal HA. The effect of prolonged administration of an anabolic steroid (oxandrolone) on growth in boys with constitutionally delayed growth and puberty. Eur J Pediatr. 1995;154(12):953-7.

19 Marin G, Domené HM, Barnes KM, Blackwell BJ, Cassorla FG, Cutler GB Jr. The effects of estrogen priming and puberty on the growth hormone response to standardized treadmill exercise and arginine-insulin in normal girls and boys. J Clin Endocrinol Metab. 1994; 79(2):537-41.

20 Penta L, Cofini M, Lucchetti L, Zenzeri L, Leonardi A, Lanciotti L, et al. Growth hormone $(\mathrm{GH})$ therapy during the transition period: should we think about early retesting in patients with idiopathic and isolated GH deficiency? Int J Environ Res Public Health. 2019;16(3):307

21 Bizzarri C, Pedicelli S, Boscherini B, Bedogni G, Cappa M, Cianfarani S. Early retesting by GHRH + arginine test shows normal GH response in most children with idiopathic GH deficiency. J Endocrinol Invest. 2015;38(4): $429-36$. 